\title{
Potential Nanomaterials and their Applications in Modern Medicine: An Overview
}

\author{
M.T.H. Bhuiyan ${ }^{1}$, M.N. Chowdhury ${ }^{2}$, Mst. Shahanaz Parvin ${ }^{1}$ \\ ${ }^{1}$ Department of Physics, Pabna University of Science and Technology, Pabna, Bangladesh \\ ${ }^{2}$ Department of Basic Sciences and Humanities, University of Asia Pacific, Dhaka, Bangladesh
}

\begin{abstract}
This paper focuses on the recent advances in nanomaterials which are playing important roles in medical science and also the nanomaterials which can be potential candidates in medicine and medical device applications. The salient properties, functional activities, advantages and disadvantages of these materials are also investigated. Nanotechnology have attracted much interest for their promising potentials in cancer treatment, targeted drug delivery and high precision medical imaging devices. Nanomaterials derived super sensitive biosensors have real potential and are greatly required in early detection of rare molecular and genetic signals associated with different diseases. Nanomaterials with enhanced magnetic (i.e., paramagnetic or super-paramagnetic) properties, high electric conductivity and well surface reactivity are highly desirable in the field of nanomedicine.
\end{abstract}

Keywords: Nanomedicine, MRI, Targeted Drug Delivery, Cancer Treatment, Gene Sequence, Tissue Repairing, Surgical Implant.

\section{INTRODUCTION}

The term nanomedicine encompasses a broad range of nanotechnologies and nanomaterials which are used in modern medicine. Nanocrystalline semiconductor particles have drawn considerable interest in recent years because of their special properties such as a large surface to volume ratio, increased activity, special electronic properties, and unique optical properties as compared to those of the bulk materials [1]. The properties of nano materials can greatly influence their interactions with biomolecules and cells, due to their unusual size, shape, chemical composition, surface structure, charge, solubility and agglomeration. Application of nanotechnology holds the promise of providing great benefits for society in the realm of biological devices, nanoelectronic biosensors, and biological machines. Functionalities can be added to nanomaterials by interfacing them with biological molecules or structures. The size of nanomaterials is comparable to that of most biological molecules and structures; therefore, nano-materials can be useful for both in vivo and in vitro biomedical research and applications. The integration of nano-materials with biology has led to the development of diagnostic devices, contrast agents, analytical tools, physical therapy applications, and drug delivery vehicles. Nanotechnology is already being used as the basis for new, more effective drug delivery systems and is in early stage development as scaffolding in nerve regeneration research. Nanomedicine could lead to breakthroughs in terms of detecting, diagnosing, and treating various forms of cancer. Nanotechnology is already moving from being used in passive structures to active structures, through more targeted drug therapies or "smart drugs". These new drug therapies have already been shown to cause fewer side effects and be more effective than traditional therapies. In the future, nanotechnology will also assist in the formation of molecular systems that may be strikingly similar to living systems. These molecular structures could be the basis for the regeneration or replacement of body parts that are currently lost to infection, accident, or disease. The rapid growth in industrial production and the attendant environmental impact have made it essential to develop highly sensitive and selective sensors for the detection of various pollutants in the atmosphere [2]. Semiconductor sensors, due to their particular advantages, such as high response, low cost, and portability, are widely used for the detection of toxic or dangerous gases and monitoring of air pollution in the environment. In this work we will study different nano composites and nanoparticles which are used in modern medicine and medical devices along with their characteristic properties and functional activities. 


\section{Applications of Nanotechnology in Medicine}

Application of nanotechnology in medicine at present being developed involves employing nanoparticles to deliver drugs, heat, light or other substances to specific types of cells (such as cancer cells). Particles are engineered so that they are attracted to diseased cells, which allow direct treatment of those cells. Nano particle are used for treatment of breast cancer and non-small-cell lung cancer (NSCLC). Nano particles are used to deliver the drug with improved effectiveness for treatment for head and neck cancer. This technique decreases damage to healthy cells in the body and allows for earlier detection of disease. In this section we have studies deferent nanomaterials and their physiochemical properties which are used in modern medicine.

\subsection{Mesoporous Silica Nanoparticles (MSNs)}

Nanoparticle based targeted drug delivery systems hold great promise for cancer therapy because of their targeting functions, sustained drug release profiles, reduced side effects, and the ability to overcome multidrug resistance $[4,5,6,7]$. A variety of materials have been exploited to meet different therapeutic needs, many with great potential for topical delivery. When compared to polymer and carbon nanotubes which have been widely investigated as carriers in drug delivery systems. Mesoporous $\mathrm{SiO}_{2}$ has the potential to serve as a versatile drug nano-carrier for smart drug delivery [8]. For example, Mesoporous $\mathrm{SiO}_{2}$ can level off the drug concentration at the targeted area as the drug molecules are released from the ordered pores by a tunable diffusion. This will reduce drug dosage and side effects [9]. Furthermore, MSNs are biocompatible, providing additional advantages for Mesoporous $\mathrm{SiO}_{2}$ to be used as a base for cancer treatment [10]. Researchers found that mesoporous $\mathrm{SiO}_{2}$ supported drug delivery system can block the notch signaling in cancer by scheming the delivery of secretase inhibitors to cancer cells and a drug delivery system with sustained-release property based on rattle-type hollow magnetic mesoporous spheres with ibuprofen loading capacity of $302 \mathrm{mg} \mathrm{g}^{-1}$ is constructed [11]. However, even though with a high surface area and a high pore volume, mesoporous $\mathrm{SiO}_{2}$ showed a relative low loading capacity of 5-fluorouracil (5-FU), a standalone therapeutic for the treatment of colon cancer, which may be due to the electrostatic repulsion between the drug and the carriers. In comparison to those carriers with a higher drug loading capacity, more MSNs were required to be taken up by cancer cells to achieve an effective cure concentration for cancer therapy. From a technical point of view, it is more desirable to deliver a lesser quantity of mesoporous $\mathrm{SiO}_{2}$ to minimize the side effects to patients [12]. Therefore, increasing the drug loading capacity of MSNs is essential for further optimizing the mesoporous $\mathrm{SiO}_{2}$ based 5FU delivery system. On the other hand, hollow MSNs (HMSNs) encapsulate more drugs than nonhollow ones as the hollow cavity supplied more space for the storage of the drugs. The capacity to encapsulate drugs in nanoparticles can be improved by functionalizing or optimizing the drug encapsulation conditions [13]. Precisely controlling the grafting of chemical groups on MSNs would result in an improvement in drug loading.

\subsection{Fe3O4 Magnetic Nanoparticle}

Magnetic nanoparticles are excellent materials in the field of biotechnology and biomedicine because they have many application including targeted drug delivery, cell leveling and magnetic cell separation, hyperthermia, tissue repairing, magnetic resonance imaging (MRI), protein purification, artificial muscle applications, radionuclide therapy, etc. However magnetic nanoparticles used in biomedicine must meet some requirements. They have to be non-toxic, chemically stable, and uniform in size, well stabilized under physiological condition, bio-compitable. Magnetite $\left(\mathrm{Fe}_{3} \mathrm{O}_{4}\right)$ and Maghemite $\left(\gamma-\mathrm{Fe}_{3} \mathrm{O}_{4}\right)$ are the most suitable iron oxide nanoparticles employed for biomedical applications because they are biocompatible, have low toxicity in the human body, and show a superparamagnetic characteristics. As for example, the injection of high concentrations $\left(c \sim 10 \mathrm{~kg} / \mathrm{m}^{3}\right)$ of magnetic nanoparticles directly into tumors at known site and irradiating them with an alternating current magnetic field caused the temperature of the tumors to increase to $43^{\circ} \mathrm{C}$. Therefore, it is known that iron oxide nanoparticles are harmless, can be selectively amassed in hidden tumors to some degree, and can be sufficiently heated when existing at high concentrations. The main advantage of magnetic nanoparticles is that the nanoparticles can reach the cancer tissue directly by travelling through the sub-micrometer spaces between blood cell walls. Therefore, for practical use, the nanoparticles should not form long chains or large clusters sample size (nm). For biomedical applications, magnetic iron oxide nanoparticles must be dispersed in bio-compatible media in order to obtain colloidal suspensions. In order to prevent the particle aggregation and to improve the 
biocompatibility and stability, nanoparticles are coated with various surfactants: poly (ethylene glycol), oleic acid, poly (acrylic acid), gluconic acid, liposome and fatty acid. Surface functionalized magnetic nanoparticles have been widely used in a range of biological applications [15-17]. Magnetite $\left(\mathrm{Fe}_{3} \mathrm{O}_{4}\right)$ is easily degradable and is useful, therefore, in bio-separation and catalytic processes magnetite nanoparticles own high surface energy and thus tend to quickly aggregate. Such strong aggregation, however, may alter their adsorption properties and magnetic efficiency; hence the nanoparticles are often coated with an organic or inorganic layer to check aggregation. Such coatings not only stabilize the magnetite nanoparticles but can easily be used for further functionalization. Several methods have already been developed by different scientists for the preparation of magnetic nanoparticles coated with a polymer, such as polyethylene glycol (PEG) and silica-containing organic material in the form of a core or shell structure, with the silica or PEG shell coated onto magnetic nanoparticles [18-19]. Coating enhances hydrophilicity and enhances biocompatibility [20-21]; the core or shell structure has a number of attractive properties, including high adsorption capacity and chemical and thermal stability [22]. As the shell provides active groups on its surface that make available binding sites for enzymes, proteins, or drugs, magnetic nanoparticles have the potential to serve as drug carriers that can selectively target cancer cells, for example, and provide controlled release of chemotherapeutics. Magnetite nanoparticles coated with amino propyl triethoxy silane have many applications as adsorbent layers for removal of aqueous heavy metals during waste water treatment. The nontoxic nature of PEG-modified nanoparticles may be useful for more efficient biotechnology application [23]. Several studies have been performed to evaluate the toxicity of magnetite nanoparticles and found surfactant-modified magnetite nanoparticles displaying negligible toxicity.

\subsection{Silver Nanoparticles (Ag-NPs)}

Various nano sized antibacterial agents such as metal, carbon, and metal oxide nanoparticles have been studied for potential in effective treatment of pathogenic microorganisms (causes for infectious diseases) [27-29]. Among the existing nano antimicrobials, silver nanoparticles (Ag-NPs) are recognized as a promising candidate to fight against pathogens. Ag-NPs are found to exhibit superior antibacterial activity and broad spectrum of antimicrobial activity against bacteria, fungi, and even viruses [30]. Because, Silver oxide show high reactivity with large surface area to volume in nanoscale range. Thus more silver can interact in body fluids to encounter and inhibit microbes. However, the Ag-NPs showed two major shortcomings for application in practice [31]: (i) particles size instability of silver nanoparticles in aqueous solution leads to aggregation of particles and results in reduction in antibacterial activity of materials and (ii) when the using high concentrations, the AgNPs could exhibit a highly toxic potential to human cells and ecology. It was shown that main reason of Ag-NPs mediated cyto-toxicity was mainly ascribed to the induction of reactive oxygen species (ROS) due to release of silver ions. Therefore, findings of novel nanostructures as alternatives were proposed by decorating Ag-NPs on supporting materials to improve antibacterial performance of materials and minimize adverse effects of silver. Recently, development of carbon-silver nanocomposites composed of carbon nanostructures decorated with silver nanoparticles demonstrates a promising approach for various technological applications such as catalyst, sensor, biomedicine, and disinfection [32]. Carbon nanotubes (CNTs) including single-walled CNTs (SWCNTs) and multiwalled CNTs (MWCNTs) have great potential as a supporting material because of their excellent properties such as high surface area and chemical stability. Especially, with the larger surface area of MWCNTs, they can be used as effective templates to deposit the Ag- NPs on the surface of MWCNTs with high stability and outstanding antimicrobial activity. Some Ag-MWCNTs nanocomposites exhibited enhanced antibacterial activities and lower cytotoxicity as compared to alone Ag-NP. The Ag-NPs-decorated MWCNTs materials shows noticeable inhibition effect with different Gram-negative bacteria (Escherichia coli and Pseudomonas aeruginosa) and Gram-positive bacteria strains (Staphylococcus aureus). Additionally, the strong adhesion of nanoparticles on functionalized MWCNTs makes the Ag-NPs less toxic because they are not released easily to the cells. MWCNTs decorated with Ag-NPs synthesized with the use of ethanol as reducing agent yielded an efficient level of antibacterial activity against Methylobacteriumspp. and Sphingomonasspp with negligible cytotoxicity toward both human and animal cells. The strong binding of silver nanoparticles with functional groups on the surface of carbon nanomaterials made them attractive with stronger bactericidal behavior and lesser toxic than that of pure silver nanoparticles due to low release to the cells. 


\subsection{Titanium Nanoparticle and it Alloys}

The most used ideal biomaterial for fabrication of surgical implants (typically used in bone replacement and dental implants) is porous titanium (Ti) thanks to its excellent physicochemical properties, biocompatibility, resistance to corrosion, high chemical stability, low toxicity and acceptability by human tissues. $\mathrm{TiO}_{2}$ also used in the photodynamic therapy for cancer treatment, drug delivery systems, cell imagine, biosensors for biological assay, and genetic engineering. Pure titanium and its alloys have high stiffness (Young's modulus) in comparison to that of the host bone, which necessitates careful implant design to ensure appropriate distribution of stresses to the adjoining bone, to avoid stress-shielding or overloading, both of which lead to bone resorption. The Young's modulus of $\mathrm{TiO}_{2}$ materials is higher than that of mineralized tissue. Moreover, the traditional structure of titanium for medical and dental implants is non-porous; this dense structure of implants can cause a mismatch between the Young's modulus of the titanium implant $(110 \mathrm{GPa})$ and natural cortical (17$20 \mathrm{GPa}$ ) and cancellous bone (around $4 \mathrm{GPa}$ ) [33,34]. Additionally, many coating and roughening techniques are used to improve cell and bone-bonding to the implant surface. To date, several studies have revealed that porous geometry may be a promising alternative to bulk structures for dental implant applications. The additive manufacturing shows promise as a technique to control pore size and shape for optimum biological properties. Most important objectives of using nano technology are aimed at optimizing the important features of interactions between the implant surface and bone tissue. Advances in orthopedic and dental implant design, as well as scaffolds for bone tissue engineering, have all contributed to the fabrication of novel porous titanium structures. Modifying the implant surface can improve the implant to bone interaction. Plasma spraying with different powder particles such as $\mathrm{Ti}_{2} \mathrm{O}$, calcium phosphate and hydroxyapatite are used to coat dental implants. Sand blasting with stiff particles such as alumina, Titanium oxide and ceramic has also been suggested to roughen the dental implant surface. Another study suggested more controllable technique of laser micro-machining of commercially pure titanium dental implant [35]. Materials with nanometer-scale porosity such as Titanium oxide nanotubes have also been used recently as implant surfacetreatments.

\subsection{Cobalt Iron Oxide (CoFe2O4) Nanoparticle}

Cobalt Iron Oxide, as a kind of magnetic nanomaterials, has long been of intensive importance in hyperthermia, cancer therapy, and molecular imaging agents in magnetic resonance imaging (MRI). The applications of $\mathrm{CoFe}_{2} \mathrm{O}_{4}$ are strongly influenced by its magnetic properties. $\mathrm{CoFe}_{2} \mathrm{O}_{4}$ nanoparticles have thin size distribution, high magnetization values, a uniform spherical shape, and superparamagnetic characteristics at room temperature which is required for biomedical applications. Ferrofluids $\left(\mathrm{CoFe}_{2} \mathrm{O}_{4}\right.$ nanoparticle) specially designed can carry drugs to specific locations in the living body through the use of applied magnetic fields. Heat treatment of organs or tissues called hyperthermia uses the property of ferrofluids of absorbing electromagnetic energy when they placed in an alternative magnetic field. This allows heating of a localized part of a living body, such as malignant tumor, up to temperature $42-46^{\circ} \mathrm{C}$ which reduces the viability of cancerous cells. For various applications, particularly in biomedicine, magnetic particles in ferrofluids, besides the requirement for small sizes, must have large enough magnetization. Depending on the relative magnitude of anisotropy energy with respect to thermal energy, the particles exhibit superparamagnetic behavior when magnetic moment of the particle overcomes the anisotropy energy barrier by thermal activation. Another potential treatment of cancer includes attaching magnetic nanoparticles to free-floating cancer cells, allowing them to be capture and carried out of the body. Magnetic nanoparticle can be used for the detection of cancer. Magnetic nanoparticle can be conjugated with carbohydrates and used for decection of bacteria. It is also used for a variety of genetics application.

The advantages of $\mathrm{CoFe}_{2} \mathrm{O}_{4}$ nanoparticles is that it can be synthesized by a co-precipitation method followed by ultra sonication and the synthesized $\mathrm{CoFe}_{2} \mathrm{O}_{4}$ nanoparticles have high surface area (140.9 $\left.\mathrm{m}^{2} \mathrm{~g}^{-1}\right)$ and small size $(2-8 \mathrm{~nm})$. It can be produced in large scale and economically inexpensive and can be recovered from the reaction mixture. The recovered catalyst can be used for multiple cycles without much loss of its activity. Thus $\mathrm{CoFe}_{2} \mathrm{O}_{4}$ emerged as an efficient, sustainable, stable and recyclable catalyst $[36,37,38]$. 


\section{ApPlications of Nanomaterials in SENSOR TeChNOLOGY}

Bio-sensors are used to incorporate living components, including tissues or cells which are electrically excitable or are capable of differentiating in to electrically excitable cells, and which can be used to monitor the presence or level of a molecule in a physiological fluid [50]. Owing to the small size of nano particles can be of great use in oncology, particularly in imaging. Nano particles, such as quantum dots, with quantum confinement properties, such as size-tunable light emission, can be used in conjunction with magnetic resonance imaging, to produce exceptional images of tumor sites. As compared to organic dyes, nano particles are much brighter and need one light source for excitation. Thus the use of fluorescent quantum dots could produce a higher contrast image and at a lower cost than organic dyes used as contrast media. But quantum dots are usually made of quite toxic elements.

\subsection{Metal Oxide Nanostructures in Electrochemical Biosensor}

Plenty of papers have been published in the literature indicating nanostructured metal oxides as a convenient component, forming an appropriate environment for the immobilization of enzyme at the electrode surface and its interaction with metallic or conducting electrode surface. Stable immobilization of macromolecular biomolecules on semiconducting metal oxide nano-surface with complete retention of their biological identification properties is a crucial problem for the commercial development of miniaturized biosensor. Owing to large specific surface area and high surface free energy of nanoparticle scan absorb enzymes strongly and play an important role in the immobilization of enzymes in construction of biosensor devices. Generally, the adsorption of enzymes directly on to naked surfaces of bulk materials may frequently result in their denaturation and loss of bioactivity. However, the adsorption of such enzymes onto the surfaces of nanoparticles can retain their bioactivity because of biocompatibile nature of metal oxides nanoparticles. Since most of the metal oxide nanoparticles carry high isoelectric point (>IEP), they can electrostatically adsorb enzymes with different charges with the low isoelectric point enzymes or protiens. Low isoelectric point enzymes and protein strongly adsorbed on the nanostructured metal oxide surface and provides direct electron communication between the enzymes and conducting electrode. Several investigators have studied the influence of nanoparticle size on the performance of the prepared biosensors and nanoparticles with smaller size were found to be more suitable for enzyme immobilization. Many similar studies have been reported for the construction of biosensors based on the immobilization of different enzymes with nanostructured metal oxides, such as glucose oxidase, cholesterol oxidase, urease, HRP, myoglobin, hemoglobin, cytochrome $\mathrm{C}$, and tyrosinase etc. Other nanostructured metaloxides, such as $\mathrm{CeO}_{2}, \mathrm{SnO}_{2}, \mathrm{Fe}_{3} \mathrm{O}_{4}, \mathrm{MnO}_{2}, \mathrm{Pr}_{6} \mathrm{O}_{11}, \mathrm{Sb}_{2} \mathrm{O}_{3}, \mathrm{TiO}_{2}, \mathrm{ZnO}$ and $\mathrm{ZrO}_{2}$ nanoparticles have been used for the immobilization of enzymes for sensitive bioelectronic device development. Electrical contacting of redox-enzymes with electrodes is a key process in the construction of third-generation enzyme electrodes. While enzymes usually lack direct electrical communication with electrodes due to the fact that the active centers of enzymes are surrounded by considerably thick insulating protein shells, and the electron transfer between electrodes and the active centers are blocked, the electro-catalytic properties of nanoparticles, mostly metal oxide nanoparticles at nano scale dimensions made them suitable for enhancing the electron transfer between the active centers of enzymes and electrodes acting as electron transfer "mediators" or "electrical wires". The conductance of metal oxides nanoparticles depend on their crystalline structure. It has been reported that the conductance of nanowires, nanotubes, nanoribbons, nanorods and nanofibers was superior in respect to other morphological nanomaterials. Due to the high electrical conductivity of the resulting dimension nanostructured metal oxides, these materials constructed sensors amplify the signal-to-noise ratio and the sensitivity by more than one order of magnitude compared to that observed at bulk materials electrodes. Modification of electrode surfaces with nanoparticles provides a micro environment similar to that of the redox proteins in native systems and gives the protein molecules more freedom in orientation, thereby reducing the insulating effect of the protein shell for direct electron transfer through the conducting tunnels of metal oxide nanoparticles. Such electrochemical biosensors could be useful for diagnosing and monitoring infectious disease, monitoring the pharmo-kinetics of drugs, detecting cancer, and disease biomarkers, analyzing breath, urine and blood for drugs of abuse [43].

\subsection{Gold (Au) Nano Particles}

Gold becomes a good catalyst for fuel cells at nanoscopic sizes and is being used in a number of nanotechnology devices for medical purposes. Positively charged gold nano particles enter the kidneys while negatively charged gold nanoparticles remained in the important organs like spleen and 
liver. The positive surface charge of the nanoparticle decreases the rate of opsonization of nanoparticles in the liver, thus affecting the excretory pathway. Due to small size of $5 \mathrm{~nm}$, nano particles can get stored in the peripheral tissues, and therefore can get collected in the body over time. Nano shells of $120 \mathrm{~nm}$ diameter, coated with gold can be used to kill cancer tumors. These nano shells are targeted to bond to cancerous cells by conjugating antibodies or peptides to the nano shell surface. Area of the tumor is irradiated with an infrared laser, which heats the gold suffciently and kills the cancer cells [29]. Gene sequencing has become more efficient with the invention of nano devices like gold nano particles, these gold particles when tagged with short segments of DNA can be used for detection of genetic sequence in a sample.

\subsection{Carbon Nanoparticles}

Carbon nano tubes, $0.5-3 \mathrm{~nm}$ in diameter and 20-1000 nm length, are used for detection of DNA mutation and for detection of disease protein biomarker. CNTs (carbon nano tubes) and DNA (de oxyribo nucleic acid) are recent candidates for new forms of nanoelectronics [51]. These are combined to create new genetically programmed self-assembling materials for facilitating the selective placement of CNTs on a substrate by functionalizing CNTs with DNA. Through recombinant DNA technology, targets labeled with distinct detectable bio markers can be defined, such as fluorescent labels, enzyme labels, and radioactive patterns, and employed in suitable biomolecular transducers [52]. After penetration of nano chains inside the tumor magnetic nanoparticles were made to vibrate by generating, radiofrequency field which resulted in the rupture of the liposome, thereby dispersing the drug in its free form throughout the tumor. When the toxic Cremophor is replaced with carbon nano particles its side effects diminished and drug targeting was much improved and needs a lower dose of the toxic paclitaxel [53].

\subsection{Cadmium Selenide}

Quantum dots, 2-9.5 $\mathrm{nm}$ in size, can help in optical detection of genes and proteins in animal models and cell assays, tumor and lymph node visualization. Cadmium selenide nano particles in the form of quantum dots are used in detection of cancer tumors because when exposed to ultraviolet light, they glow. The surgeon injects these quantum dots into cancer tumors and can see the glowing tumor, thus the tumor can easily be removed.

\section{DisCuSSION AND CONCLUSION}

Nanotechnology has attracted much attention and have shown promising growth during the last decade based on their competency in various applications particularly in cancer treatment, targeted drug delivery, antibacterial agents, sensing, and coating. However, a few key challenges must be addressed and resolved to realize the potential of nanomedicine regarding biocompatibility, toxicity and environmental hazard, etc. From the various nanocomposites developed to date, Magnetite $\left(\mathrm{Fe}_{3} \mathrm{O}_{4}\right)$, Maghemite $\left(\gamma-\mathrm{Fe}_{3} \mathrm{O}_{4}\right)$, Cobalt Iron Oxide $\left(\mathrm{CoFe}_{2} \mathrm{O}_{4}\right)$, Titanium Oxide $\left(\mathrm{T}_{2} \mathrm{O}\right)$ and it alloys, Silver, Mesoporous Silica, Cerium oxide $\left(\mathrm{CeO}_{2}\right)$, Stannum oxide $\left(\mathrm{SnO}_{2}\right)$, Manganese oxide $\left(\mathrm{MnO}_{2}\right)$, Praseodymium oxide $\left(\mathrm{Pr}_{6} \mathrm{O}_{11}\right)$, Antimony oxide $\left(\mathrm{Sb}_{2} \mathrm{O}_{3}\right)$, Zinc oxide $(\mathrm{ZnO})$ and Zirconium oxide $\left(\mathrm{ZrO}_{2}\right)$ nanoparticles are the most crucial nanoparticles and they have remarkable contribution in modern medicine. In our present study we categories these nanomaterials into three different groups in terms of their potential applications in medical science, namely, in disease treatment, surgical implant and device sensor especially in imaging technology. From our study it is clearly evident that super-paramagnetic nanocomposites (such as Magnetite $\mathrm{Fe}_{3} \mathrm{O}_{4}$ and Maghemite $\gamma-\mathrm{Fe}_{3} \mathrm{O}_{4}$ ) are hugely important in targeted drug delivery, tissue repairing, magnetic resonance imaging (MRI), protein purification, artificial muscle applications, radionuclide therapy hyperthermia, cancer therapy, and molecular imaging agents in magnetic resonance imaging (MRI). The applications of Magnetic nanoparticles are biocompatible and can be selectively accumulated in hidden tumors by travelling through the sub-micrometer spaces between blood cell walls at high concentrations, and can be adequately irradiated using AC magnetic field without damaging healthy tissues. It is also evident that Titanium oxide nanoparticle and it alloys are promising candidates in bone replacement and dental implants, photodynamic therapy for cancer treatment, drug delivery systems, cell imagine, biosensors for biological assay, and genetic engineering thanks to its excellent physicochemical properties, biocompatibility, resistance to corrosion, high chemical stability, low toxicity and acceptability by human tissues. One earlier study claim that Ag-NPs are found to exhibit superior antibacterial activity and broad spectrum of antimicrobial activity against bacteria, fungi, and even viruses. Some reports 
have discussed that Mesoporous silica nano particles (MSNs) have potential to serve as a versatile drug nano-carrier for smart drug delivery (i.e., targeted drug delivery) system. Owing to large specific surface area and high electrical conductivity of biocompatible nano structured metal oxide can absorb enzymes strongly and play an important role in the immobilization of enzymes in construction of biosensor devices. Such electrochemical biosensors could be useful for diagnosing and monitoring infectious disease, monitoring the pharmo-kinetics of drugs, detecting cancer, and disease biomarkers, analyzing breath. Some reports evident silver and gold nano particles are of prime importance for drug delivery, especially in treatment of cancer and also in biosensors. Gene sequencing has become more efficient with the invention of nano devices like gold nano particles, these gold particles when tagged with short segments of DNA can be used for detection of genetic sequence in a sample. Silicon chips that are capable of implantation within the human body may permit interfacing semiconductor devices with living tissues. This paper also reveals that Gadolinium labeled nanoparticles such as carbon nanotubes, superparamagnetic iron oxides, iodine, bismuth, or gold are highly sophisticated nano-sized contrast agents are used in Computed Tomography (CT) scan and Magnetic Resonance Imaging (MRI) technology. Quantum dots semiconducting nanocrystals (i.e., Cadmium Selenide) emit a wide spectrum of bright colors when they are illuminated with ultraviolet light. Therefore, quantum dots provide excellent contrast for fluorescent imaging to locate and identify specific kinds of cells and biological activities. Quantum dots are also excellent tools for delivery of gene or drugs into stem cells. Nanotechnology allows us to create materials and coatings to construct more and more advanced modern medicine relying on electronic devices that are fully biocompatible, so that they integrate seamlessly with the body's systems. Implantable drug delivery systems can deliver small amounts of drugs on a regular basis, so that the patient does not need to be injected. Implantable drug delivery systems give a more consistent drug level in the blood compared to injections, which often makes the treatment more effective and reduces side effects. By using active monitoring capabilities built into the device, the dosage can be adjusted to suit changes in physical activity, temperature changes and other variables. Besides the numerous advantages of nanoparticles in medicine, it has some demerits too. As for instance, some nanoparticles are highly toxic, not biocompatible, instability, particle aggregation. Moreover, bio-distribution of these nanoparticles is still imperfect due to the complex host's reactions to nano- and micro sized materials and the difficulty in targeting specific organs in the body. Fortunately, all these problems are solvable and nano coating could be a good technique to overcome these problems.

\section{REFERENCES}

[1] Henglein, "Small-particle research: physicochemical properties of extremely small colloidal metal and semiconductor particles," Chemical Reviews, vol. 89, no. 8, pp. 1861-1873, 1989.

[2] Gas Semiconducting Sensors Based on Metal Oxide, Nanocomposites L. I. Trakhtenberg, G. N. Gerasimov, V. F. Gromov\& T. V. Belysheva, O. J. Ilegbusi.

[3] Nanostructured Metal Oxides Based Enzymatic Electrochemical Biosensors, Anees A. Ansari, M.Alhoshan, M.S. Alsalhi and A.S. Aldwayyan.

[4] J. M. Rosenholm, A. Meinander, E. Peuhu et al., "Targeting of porous hybrid silica nanoparticles to cancer cells," ACS Nano, vol.3, no.1, pp.197-206, 2009.

[5] X. W. Lou, L. A. Archer, and Z. Yang, "Hollow micro-/nanostructures: synthesis and applications," AdvancedMaterials,vol. 20, no. 21, pp. 3987-4019, 2008.

[6] Z. Ma, J. Bai, Y. Wang, and X. Jiang, "Impact of shape and pore size of mesoporous silica nanoparticles on serum protein adsorption and RBCS hemolysis," ACS Applied Materials and Interfaces, vol.6, no.4, pp.2431-2438, 2014.

[7] W.Zhao,H.Chen,Y.Li,A.Li,M.Lang,andJ.Shi, "Uniformrattle-type hollow magnetic mesoporous spheres as drug delivery carriers and their sustained-release property," Advanced Functional Materials, vol. 18, no. 18, pp. 2780-2788, 2008.

[8] M. Sardan, A. Yildirim, D. Mumcuoglu, A. B. Tekinay, and M.O. Guler, "Noncovalent functionalization of mesoporous silica nanoparticles with amphiphilic peptides," Journal of Materials Chemistry B, vol.2, no.15, pp.2168-2174, 2014.

[9] N. H. N. Kamarudin, A. A. Jalil, S. Triwahyono et al., "Role of 3-amino propyl triethoxy silane in the preparation of mesoporoussilica nanoparticles for ibuprofen delivery: effect on physicochemical properties," Microporous and Mesoporous Materials, vol. 180, pp. 235-241, 2013. 
[10] Pros and Cons on Magnetic Nanoparticles Use in biomedicine and biotechnologies application, Florina, M. Bojin and Virgil paunescu, pp 103-135, 2014.

[11] G. Paul, T. B. Frigo, J. Y. Groman, and E. V. Groman, "Synthesis of ultra small superparamagnetic iron oxides using reduced polysaccharides," Bio-conjugate Chemistry, vol. 15, no. 2, pp. 394-401, 2004.

[12] J. Namanga, J. Foba, D. T. Ndinteh, D. M.Yufanyi, and R. W. M. Krause, "Synthesis and magnetic properties of a superparamagnetic nanocomposite "pectin-magnetite nanocomposite'," Journal of Nanomaterials, vol. 2013, Article ID 137275, 8 pages, 2013.

[13] R. Vestal and Z. J. Zhang, "Synthesis and magnetic characterization of mn and Co spinel ferritesilica nanoparticles with tunable magnetic core," Nano Letters, vol. 3, no. 12, pp. 1739-1743, 2003.

[14] T.-J.Yoon, K. N. Yu, E. Kim et al., "Specific targeting, cell sorting, and bioimaging with smart magnetic silica core-shell nanomaterials," Small, vol. 2, no. 2, pp. 209-215, 2006.

[15] S. M. Moghimi, A. C. Hunter, and J. C. Murray, "Long-circulating and target-specific nanoparticles: theory to practice," Pharmacological Reviews, vol. 53, no. 2, pp. 283-318, 2001.

[16] W. Wu, Q. He, and C. Jiang, "Magnetic iron oxide nanoparticles: synthesis and surface functionalization strategies," Nanoscale Research Letters, vol. 3, no. 11, pp. 397-415, 2008.

A. K. Gupta and S. Wells, "Surface-modified super-paramagnetic nanoparticles for drug delivery: preparation, characterization, and cytotoxicity studies," IEEE Transactions on Nanobioscience, vol. 3, no. 1, pp. 66-73, 2004.

[17] M. S. A. Darwish, U. Kunz, and U. Peuker, "Preparation and catalytic use of platinum in magnetic core/shell nanocomposites," Journal of Applied Polymer Science, vol. 129, no. 4, pp. 1806-1811, 2013.

[18] W. Stöber, A. Fink, and E. Bohn, "Controlled growth of monodisperse silica spheres in the micron size range," Journal of Colloid And Interface Science, vol. 26, no. 1, pp. 62-69, 1968.

[19] M. Rai, A. Yadav, and A. Gade, "Silver nanoparticles as a new generation of antimicrobials," Biotechnology Advances,vol.27,no. 1, pp. 76-83, 2009.

[20] X.Zhang,H.Niu,J.Yan,andY.Cai,"Immobilizing silver nanoparticles onto the surface of magnetic silica composite to prepare magnetic disinfectant with enhanced stability and antibacterial activity," Colloidsand Surfaces A: Physicochemical and Engineering Aspects,vol.375,no.13,pp.186-192,2011.

[21] S.Liu,T.H.Zeng,M.Hofmannetal., "Anti bacterial activity of graphite, graphite oxide, graphene oxide, and reduced grapheme oxide: membrane and oxidative stress," ACS Nano,vol.5,no.9,pp. 6971-6980, 2011.

[22] Q.H.Tran,V.Q. Nguyen, and A.-T.Le, "Silver nanoparticles: synthesis, properties, toxicology, applications and perspectives," Advances in Natural Sciences: Nanoscience and Nanotechnology, vol.4, no.3, Article ID 033001, 2013.

[23] R. Prucek, J. Tu cek, M. Kilianov' a et al., "The targeted antibacterial and antifungal properties of magnetic nanocomposite of iron oxide and silver nanoparticles," Biomaterials,vol.32,no.21, pp. 4704-4713, 2011.

[24] W.-P. Xu, L.-C.Zhang, J.-P. Li et al., "Facile synthesis of silver graphene oxide nanocomposites and their enhanced antibacterial properties," Journal of Materials Chemistry, vol.21, no.12, pp. 4593-4597, 2011.

[25] G. Olmedo, G. Duff' o, R. L. Cabrini, and M. B. Guglielmotti, "Local effect of titanium implant corrosion: an experimental study in rats," International Journal of Oral and Maxillofacial Surgery, vol.37, no.11, pp.1032-1038, 2008.

[26] R. M. Wazen, L.-P. Lefebvre, E. Baril, and A. Nanci, "Initial evaluation of bone ingrowth into a novel porous titanium coating," Journal of Biomedical Materials Research, PartB: Applied Biomaterials, vol.94, no.1, pp.64-71, 2010.

[27] Magnetic and Structural Studies of $\mathrm{CoFe}_{2} \mathrm{O}_{4}$ Nanoparticles Suspended in an Organic Liquid Branka Babi T-Stoji, Vukoman Jokanovi, Dušan Milivojevi, ZvonkoJagliIi, Journal of Nanomaterials, Volume 2013, Article ID 741036, 9 pages

[28] Synthesis and Magnetic Properties of Nearly Monodisperse $\mathrm{CoFe}_{2} \mathrm{O}_{4}$ Nanoparticles Through a Simple Hydrothermal Condition, Xing-Hua Li, Cai-Ling Xu, Xiang-Hua Han, Liang Qiao, Nanoscale Res Lett (2010) 5:1039-1044, DOI 10.1007/s11671-010-9599-9 
[29] Magnetic properties of $\mathrm{CoFe}_{2} \mathrm{O}_{4}$ nanoparticles synthesized througha block copolymer nanoreactor route, Sufi R. Ahmed, S. B. Ogale, Georgia C. Papaefthymiou, Ramamoorthy Ramesh, Peter Kofinas, Applied physics letters volume 80, number 9, 4 march 2002.

[30] Tomchenko, A.A.; Harmer, G.P.; Marquis, B.T.; Allen, J.W. "Semiconducting metal oxide sensor array for the selective detection of combustion gases", Sens. Actuat. B 2003, 93, 126-134.

[31] Kanan, S.M.; El-Kadri, O.M.; Abu-Yousef, I.A.; Kanan, M.C. "Semiconducting metal oxide based sensors for selective gas pollutant detection" Sensors2009, 9, 8158-8196.

[32] Kohl, D. "Surface processes in the detection of reducing gases with $\mathrm{SnO}_{2}$-based devices", Sens. Actuat 1989, 18, 71-113.

[33] "Nanostructured Metal Oxides Based Enzymatic Electrochemical Biosensors", Anees A. Ansari, M.Alhoshan, M.S. Alsalhi and A.S. Aldwayyan.

[34] M. Snure, D. Kumar, and A. Tiwari, "Progress in $\mathrm{ZnO}$ based diluted magnetic semiconductors," JOM, vol. 61, no. 6, pp. 72-75, 2009.

[35] M. Willander, L. L. Yang, A. Wadeasaet al., "Zinc oxide nanowires: controlled low temperature growth and some electrochemical and optical nano-devices," Journal of Materials Chemistry, vol. 19, no. 7, pp. 1006-1018, 2009.

[36] Adler JP 1966, Chemotaxisin bacteria Science,153708-16.

[37] Freitas RAJr 2006, Pharmacytes: an ideal vehicle for targeted drug delivery, J.Nanosci.Nanotechnol.62769-75.

[38] MutohK, T sukahara S, Mitsuhashi J, Katayama Kand Sugimoto Y 2006, Estrogen-mediated post transcriptional down-regulationo fP - glycoprotein in MDR1-transduced human breast cancer cells, Cancer Sci.971198-204.

[39] Artemov D, Solaiyappan Mand Bhujwalla Z M 2001, Magnetic resonance pharmaco angiography to detect and predict chemotherapy delivery to solid tumors, Cancer Res. 61303944.

[40] Sih H J 2006, Implantable biosensor US Patent Specification, 20060234369.

[41] Dubin V M 2006, Nano fabrication using carbon nanotubes and DNA US Patent Specification, 7122461.

[42] Grieninger G, Fu Y, Cao Y, Ahadi M Zand Kudryk B 2000, Monospecific antibodies against a sub unit of fibrinogen US Patent Specification, 6025148 (Alexandria,Virginia,USA).

[43] Villar ID, Matias IR, Arregui FJ and Claus RO 2005 ESA-basedin-fiber nanocavity for hydrogen peroxide detection, IEEE Trans. Nanotechnol. 4187-93.

[44] M. A. Rahman, Md. Tofajjol Hoseen Bhuiyan, M. M. Rahman, M.N. Chowdhury, Comparative Study of Absorbed Doses in Different Phantom Materials and Fabrication of a Suitable Phantom, Volume 3, No 1/2016, ISSN 2313-0008 (Print); ISSN 2313-0016 (Online). 